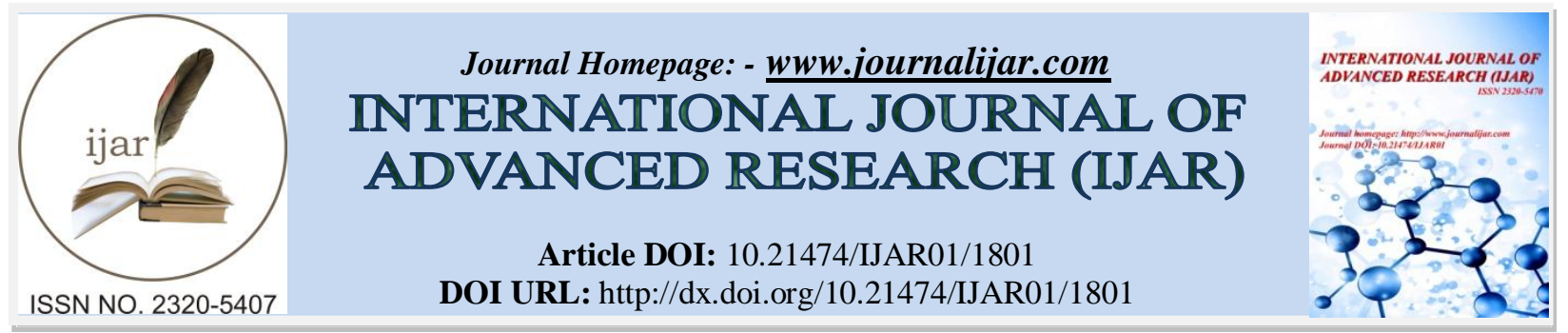

RESEARCH ARTICLE

\title{
THE IMPACT OF HIGH-INTENSITY FOCUSED ULTRASOUND ON THE HYDATID ECHINOCOCCUS CYST (EXPERIMENT IN VITRO).
}

\author{
S. Imankulov, A. Baygenzhin, K. Rustemova, I. Tashev, G. Fedotovskikh, G. Shaimardanova, N. \\ Zhampeissov, M. Erlan. \\ National Scientific Medical Research center, Astana, Kazakhstan.
}

\section{Manuscript Info}

Manuscript History

Received: 16 August 2016

Final Accepted: 19 September 2016

Published: October 2016

Key words:-

liver echinococcosis, the high-intensity

focused ultrasound, destruction of a

parasitic cyst, morphology.

\section{Abstract}

Liver echinococcosis residents of Kazakhstan is still a serious illness, hard to treatment. Selection and search for the most effective treatments for this disease is relevant today actually. The authors of the impact of high-intensity fu different effects on scolexes echinococcus been experimentally studied. Morphological studies have confirmed they have developed a technique of high-impact lowfrequency ultrasound on parasitic cysts.

Copy Right, IJAR, 2016,. All rights reserved.

\section{Introduction:-}

Echinococcosis is a severe chronic parasitic disease with an extensive, often systemic pathology, leading to disability and death of the patients. $[1,2,3,4]$.

The introduction of high-intensity focused ultrasound (HIFU-ablation) during the treatment of echinococcosis of the liver [5] undoubtedly required morphological assessment of the hydatid Echinococcus cyststate after the highintensity focused ultrasound exposure.

\section{Objective:-}

The objective of the experimental part of the study was to investigate the impact of high-intensity focused ultrasound of different dose rates on the hydatid Echinococcus larvocyst structures at the light-optical morphological level.

Experimental protocol:-

The unexposed to high-intensity focused ultrasound Echinococcus cyst of $4.4 \mathrm{~cm}$ diameter was intraoperatively removed, according to Figure 1. 


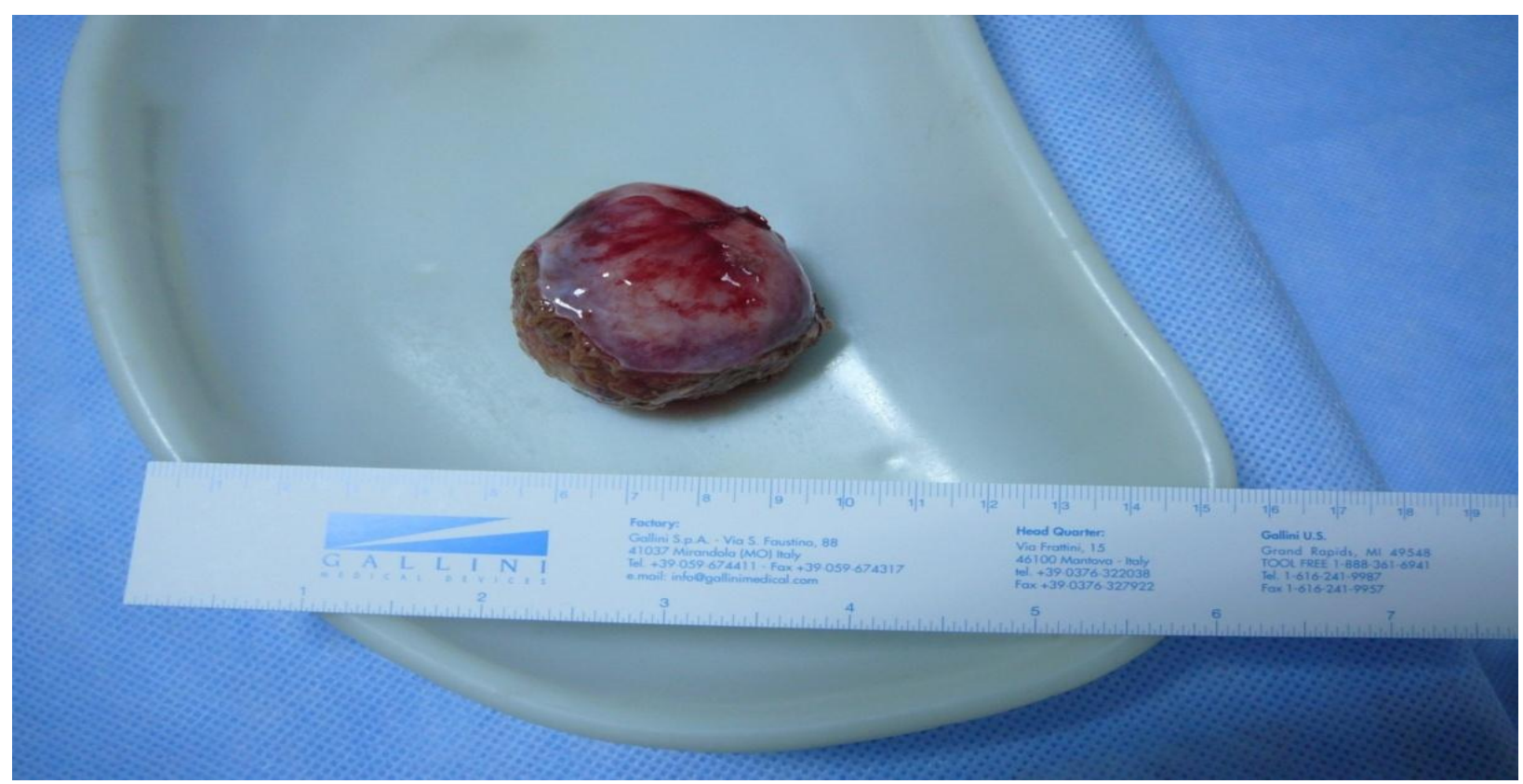

Figure 1:- The Echinococcus cyst.

The fibrous and chitinized (cuticular + germinal) envelopes were subsequently separated according to Figure 2 and Figure 3.

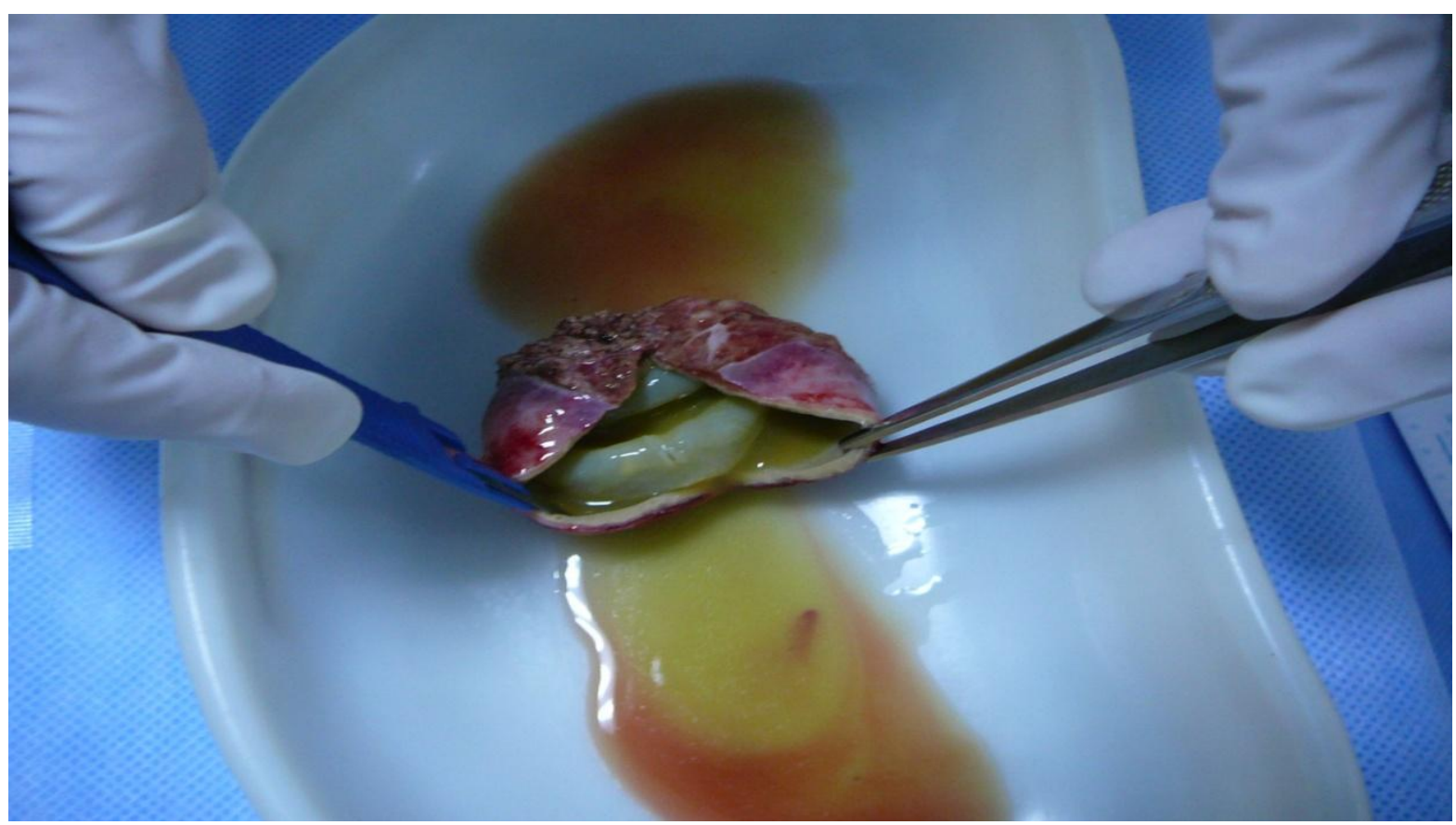

Figure 2:- Episode of the cyst preparation. 


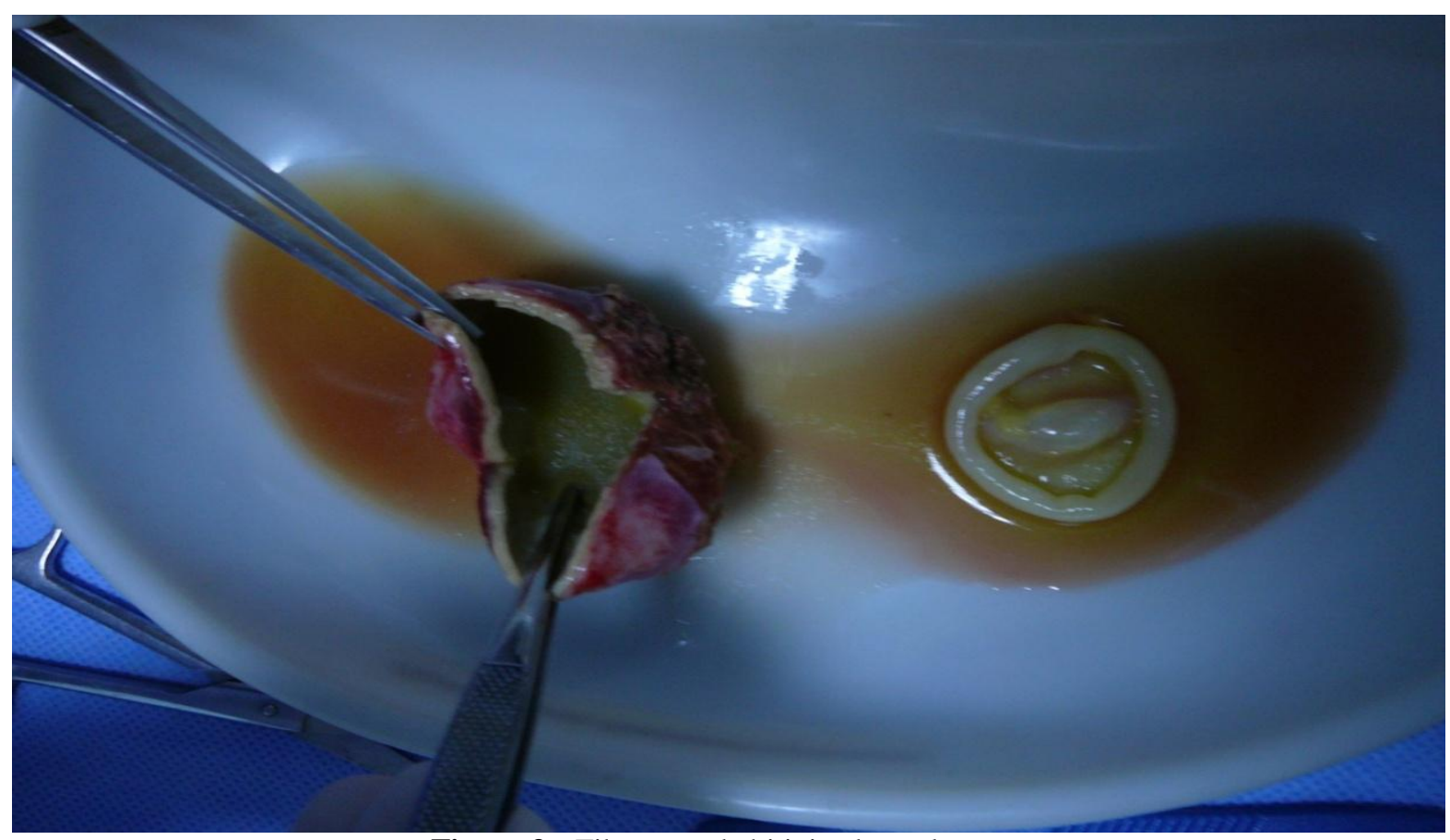

Figure 3:- Fibrous and chitinized envelopes.

The fibrous and chitinized envelopes were fragmented into 5 flaps each of $0.4 \mathrm{~cm} 2$. The material has been placed into 5 polyvinylchloride containers. (Figure5).

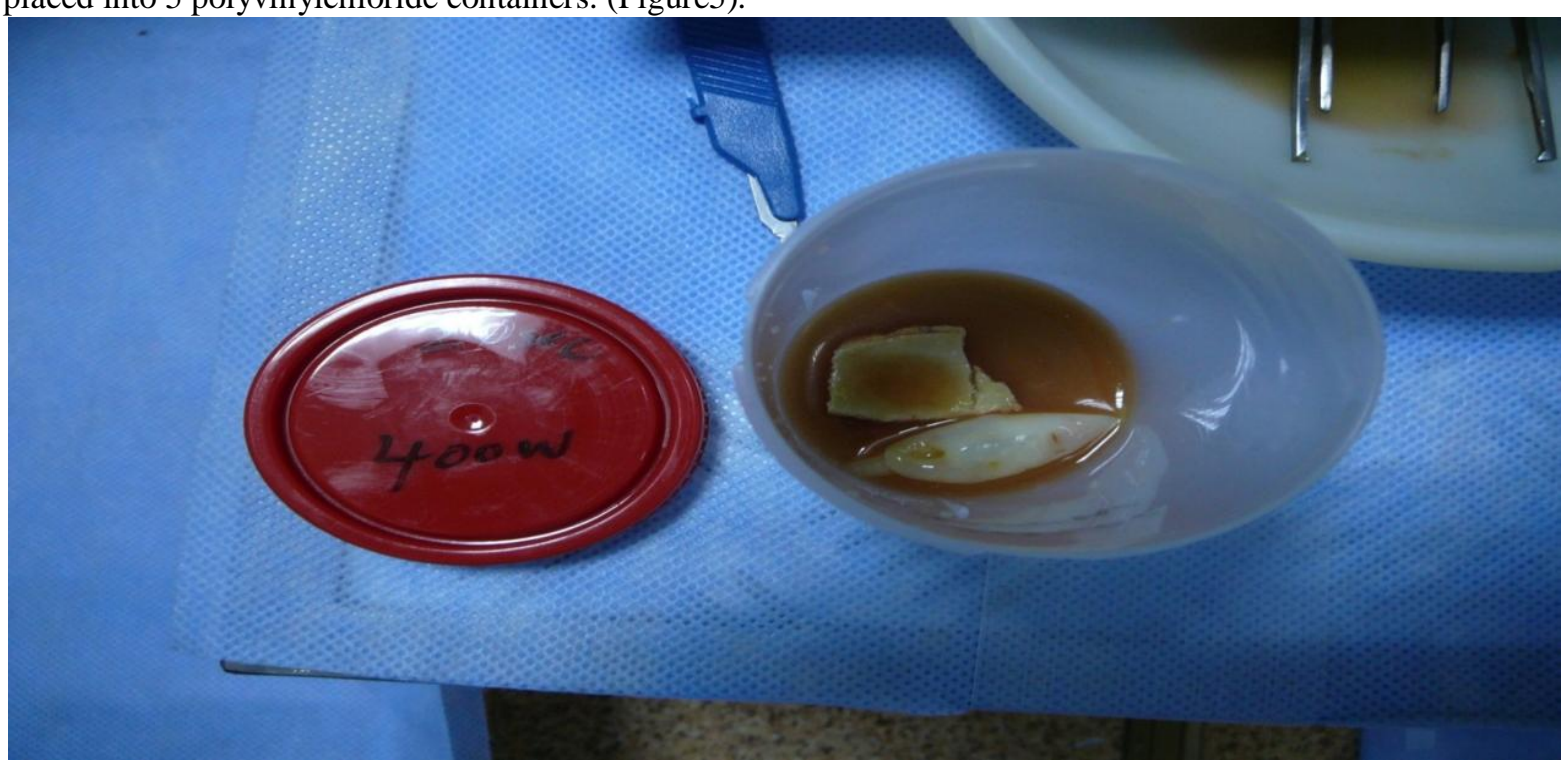

Figure 4:- Fragments of fibrous and chitinized envelopes.

One of the containers was intended for the control study, the second one for the study after the HIFU exposure with the dose rate of $100 \mathrm{~W}$, the third one for the study after the HIFU exposure with the dose rate of $200 \mathrm{~W}$, the fourth one for the study after the HIFU exposure with the dose rate of $300 \mathrm{~W}$, and the fifth one for the study after the HIFU exposure with the dose rate of 400W. (Figure.5)

The ablation by high-intensity focused ultrasound was performed using the JC system (Chongqing HIFU Technology Company, China) with a focusing lens of $21.5 \mathrm{~cm}$ diameter, $1.0 \mathrm{MHz}$ emission frequency, 16.2 $\mathrm{cm}$ focal distance. 


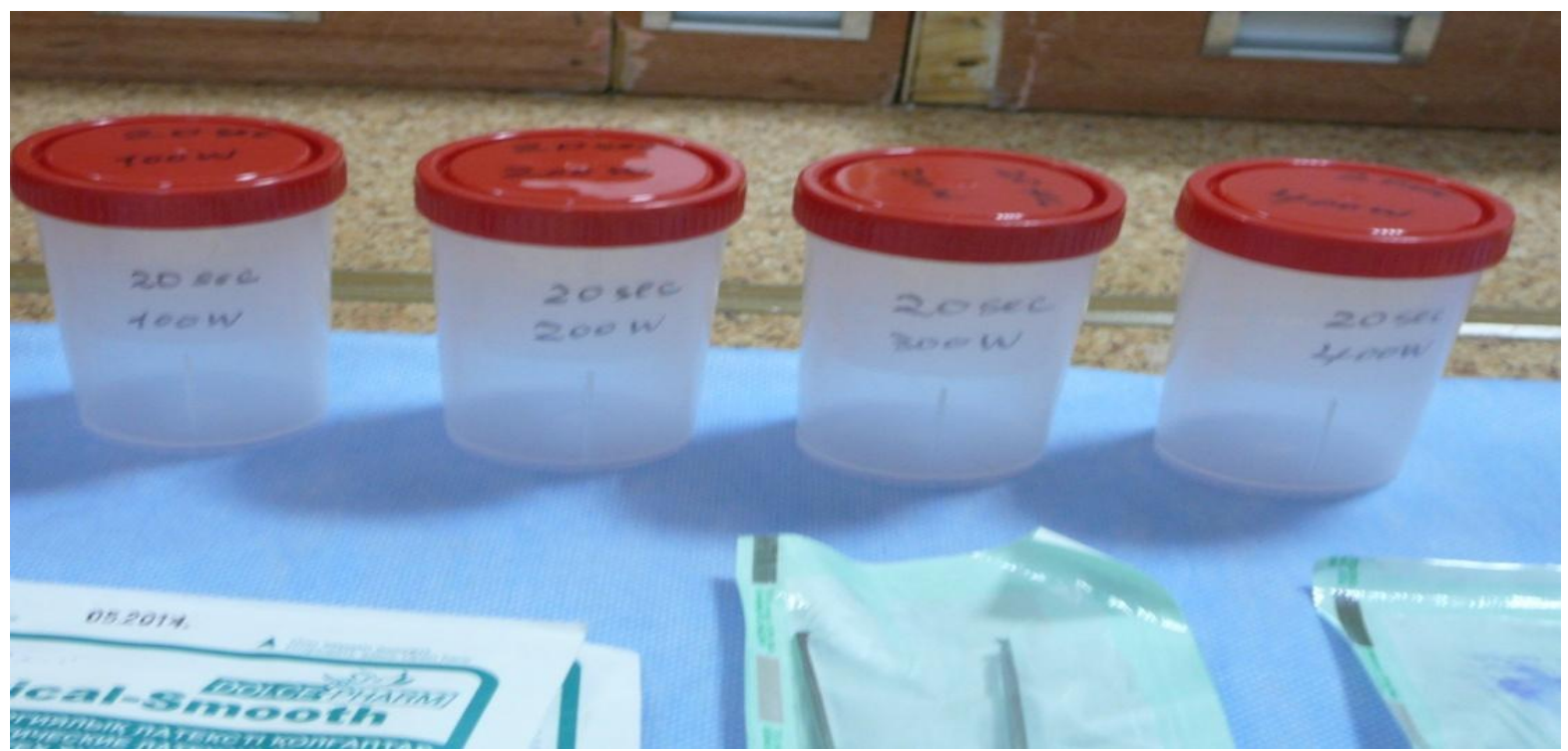

Figure 5:- Marked containers for the HIFU - therapy.

Container with the fragments of fibrous and chitinized envelopes was mounted into a frame for the test (experimental) material in accordance with Figure 6.

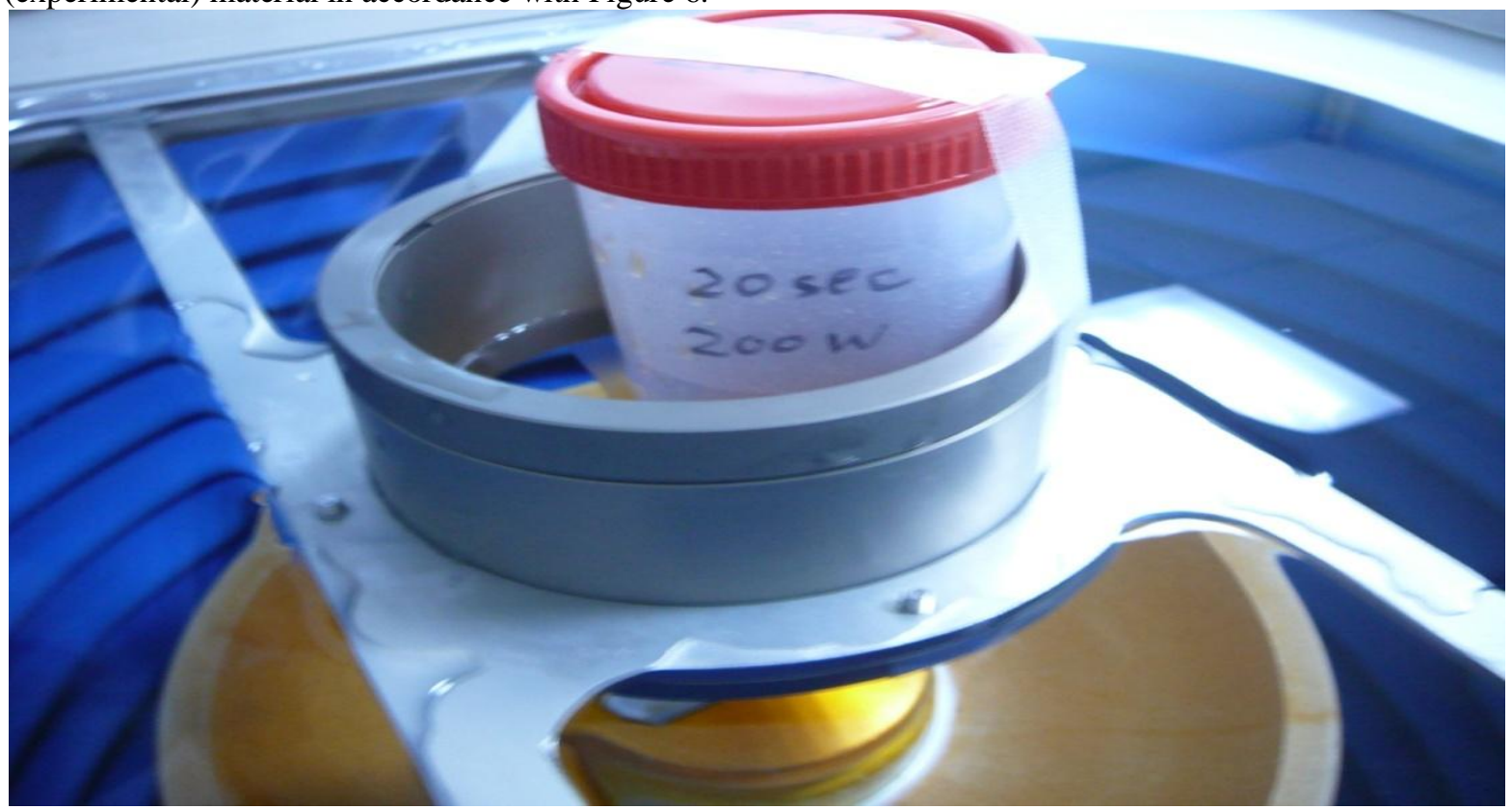

Figure 6:- Container with the material in the frame for the test control.

The HIFU exposure was 10 seconds for each fragment of the envelopes with the defined rates: 100 - 200 - 300 $-400 \mathrm{~W}$ 


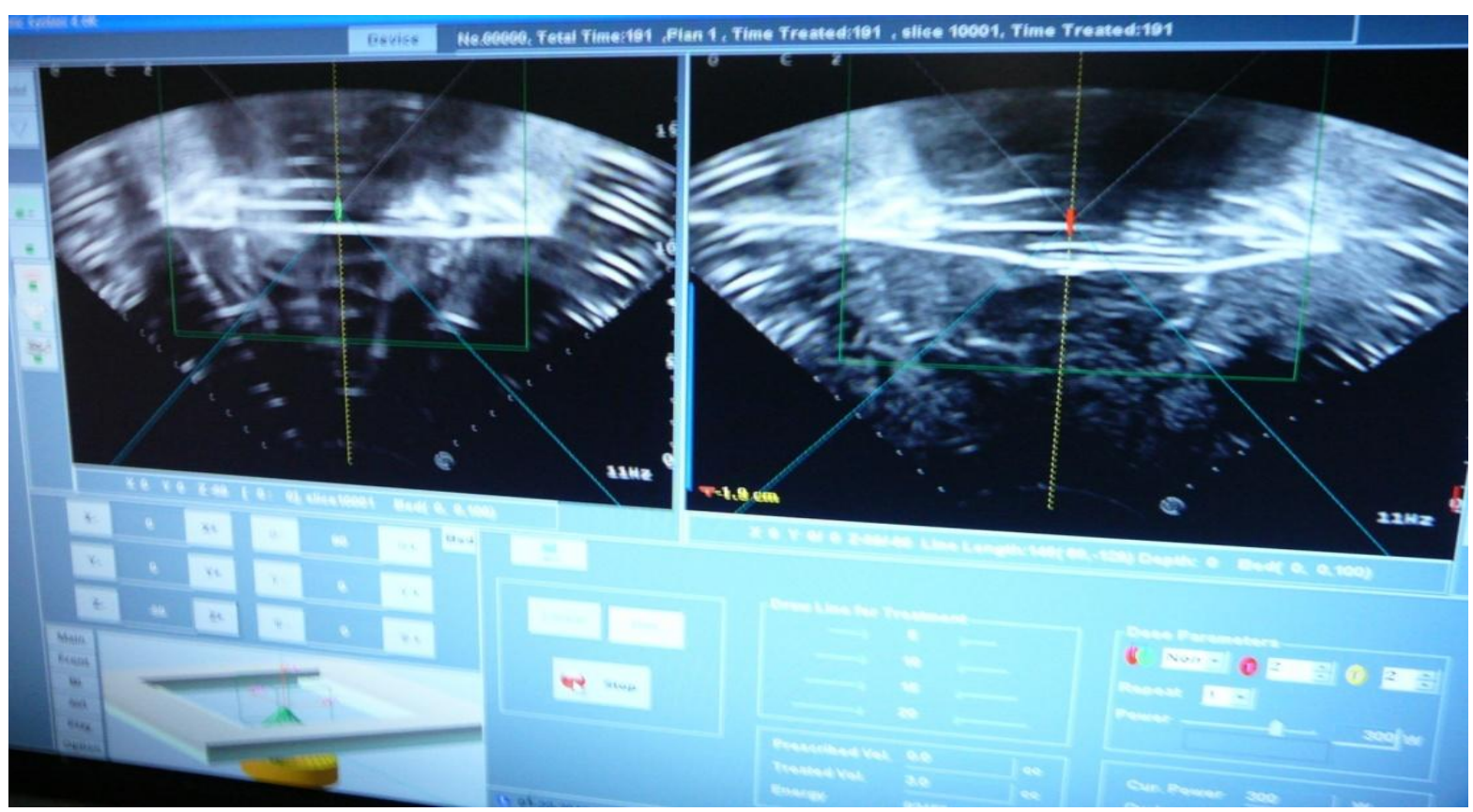

Figure 7:- The real-time HIFU therapy with rate of 300W.

\section{Histological research methods:-}

For light microscopy biopsy pieces of the wall echinococcus cyctwere fixed in $10 \%$ neutral formalin and carried out by the conventional method. Histological sections were stained with hematoxylin and eosin .

\section{Rezults:-}

The morphological study of the hydatid Echinococcus mature larvocyst wall histological sections in the control area (unexposed to the HIFU therapy) has shown that the fibrous capsule had a non-uniform thickness and was represented by the coarse-fibred and granulation tissue with fine blood vessels and foci of inflammatory infiltration in accordance with Figure 8.

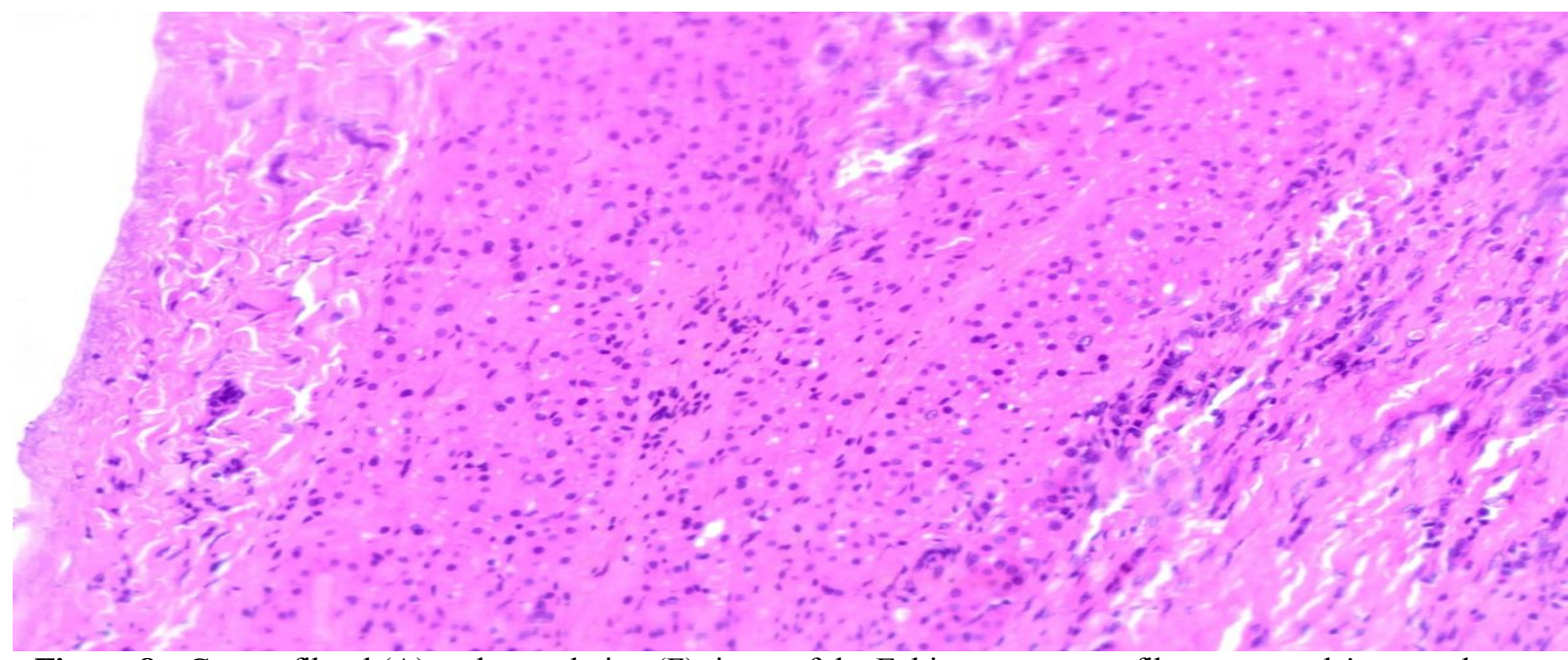

Figure 8:- Coarse-fibred (A) and granulation (Б) tissue of the Echinococcus cyst fibrous capsule's control section. Tinction by hematoxylin and eosin. Magnification x 100 
The chitinized envelope has normal layered structure according to Figure 9.

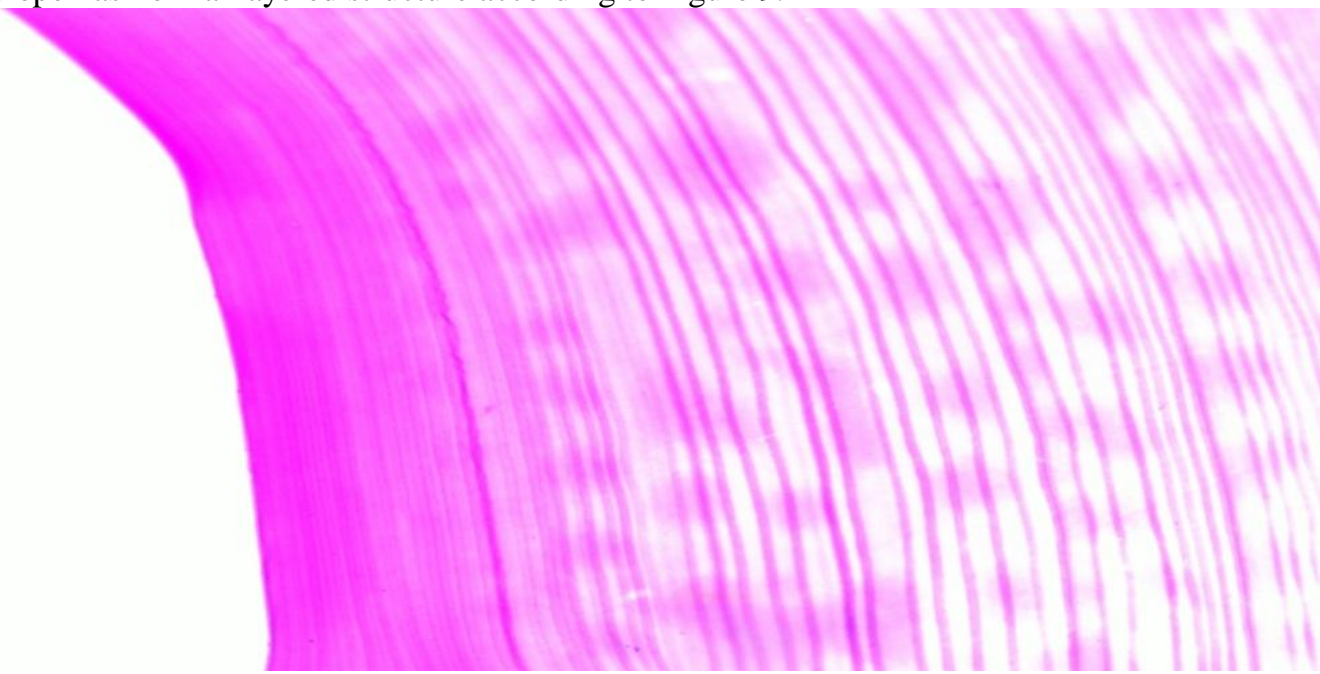

Figure 9:- The chitinized envelope of the Echinococcus cyst capsule on control section.

Tinction by hematoxylin and eosin. Magnification x 100 .

The germinal envelope consists of tegument, glycogen- and calcium-containing cells. The protoscolexes, located directly in the germinal envelope, were formed by assembly of early and postmitotic cells according to Figure 10.

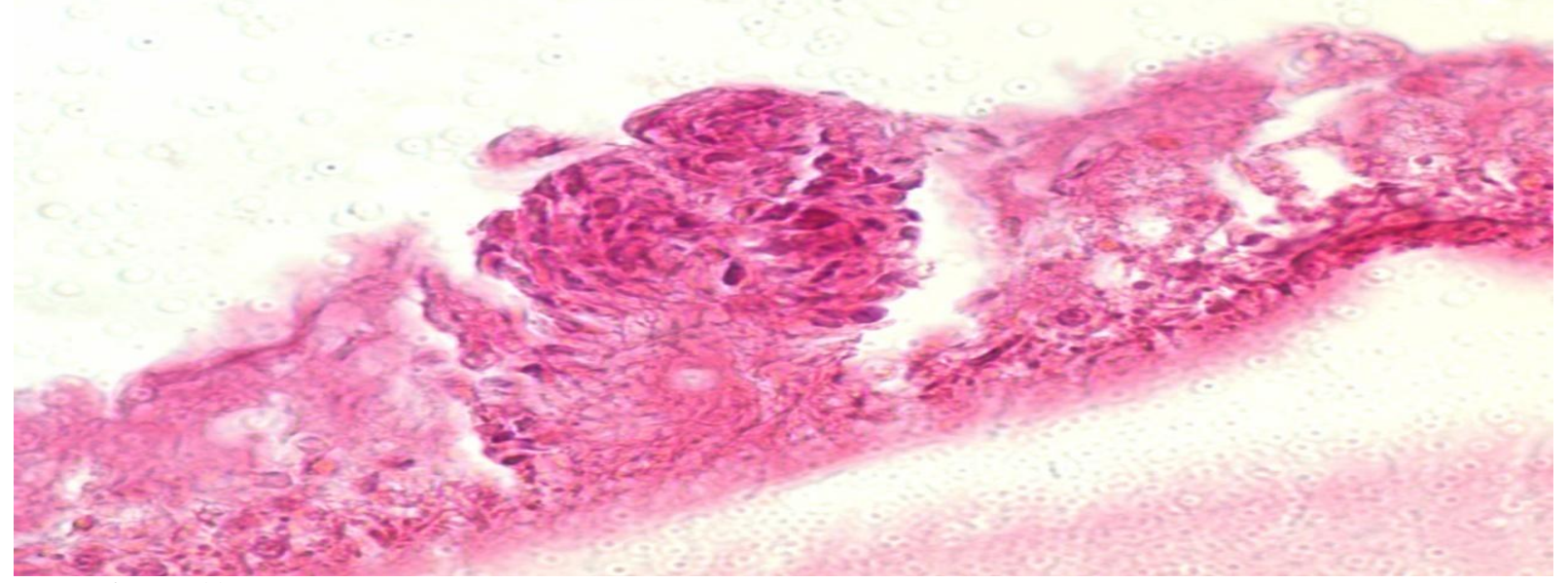

Figure 10:- Protoscolex in germinal envelope of the Echinococcus cyst capsule on control section. Tinction by hematoxylin and eosin. Magnification $\mathrm{x} 100$.

$1 \mathrm{We}$ observed obvious morphological changes of the larvocyst wall only with the exposure of $200 \mathrm{~W}$. 
Wherein, the germinal envelope of capsule partially or completely separated from the chitinized envelope, according to Figure 11.

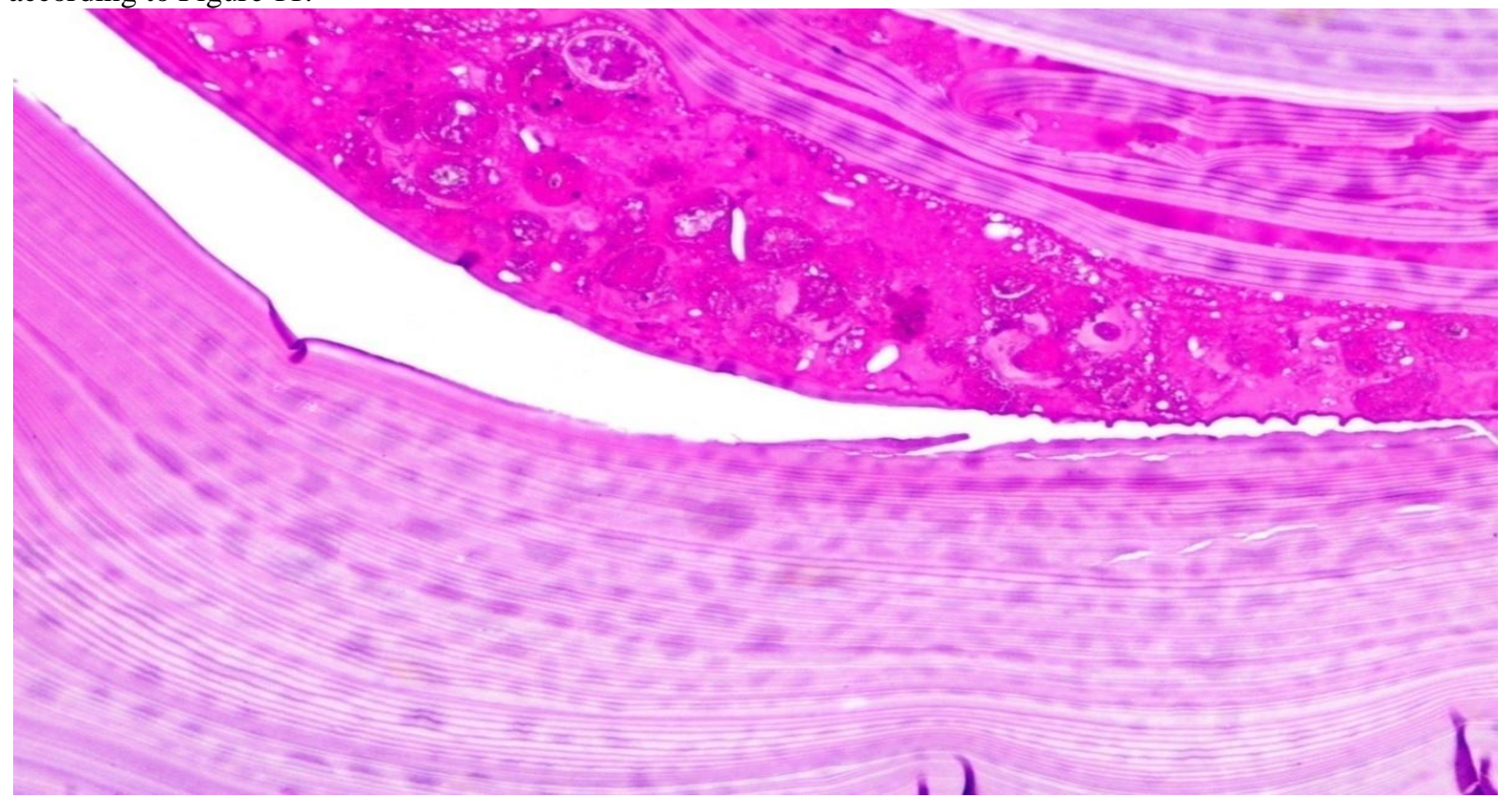

Figure 11:- Separation of germinal envelope from the chitinized one under the HIFU therapy exposure of rate of 200W.The protoscolexes structure has been distorted and destroyed. Hooklets has been disrupted.

Histologic section. Tinction by hematoxylin and eosin.Magnification x 100 .

The protoscolexes structure has been distorted and destroyed. A special feature was represented by reversed and disrupted hooklets. A part of germinal envelope was completely destroyed according to Figure 12.

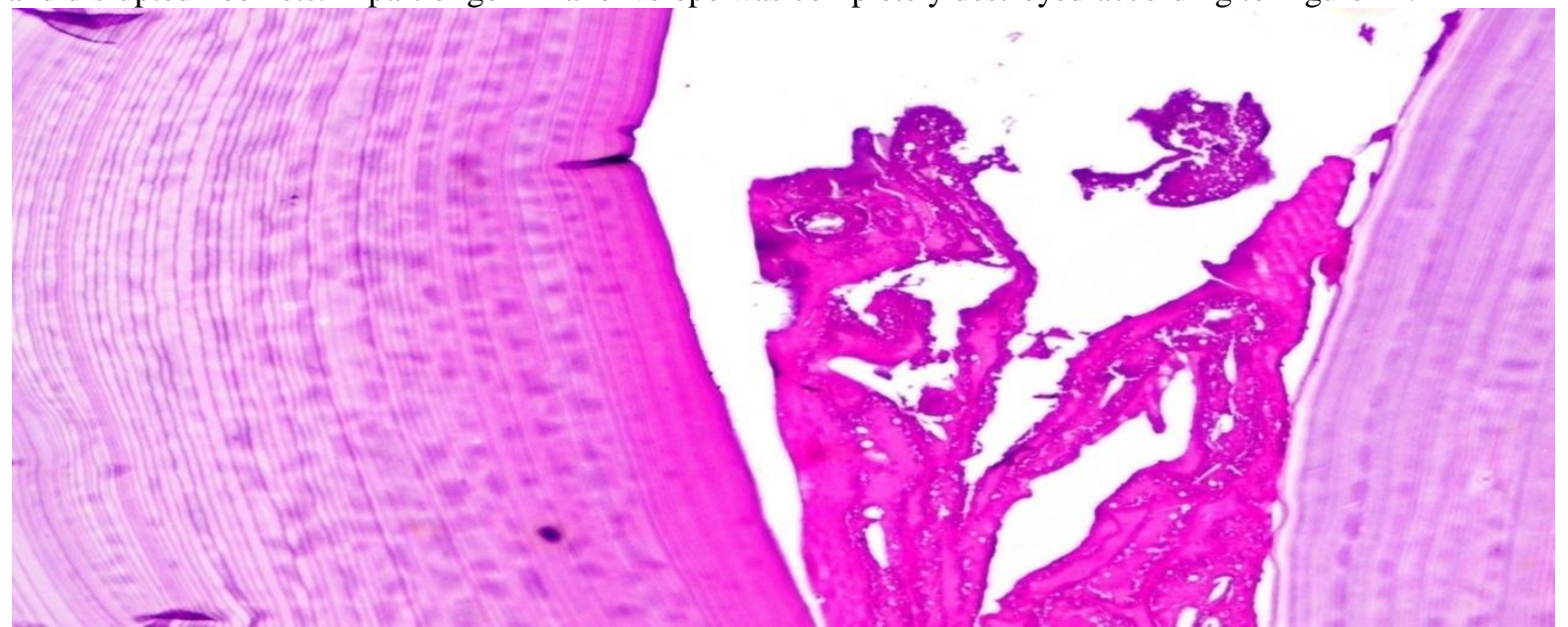

Figure 12 - Ablation of germinal envelope by the HIFU therapy exposure of the dose rate of $200 \mathrm{~W}$. Tinction by hematoxylin and eosin. Magnification x 100 .

The fibrous envelope has been loosened, the hemorrhage and destruction foci have been marked according to Figure 13. 


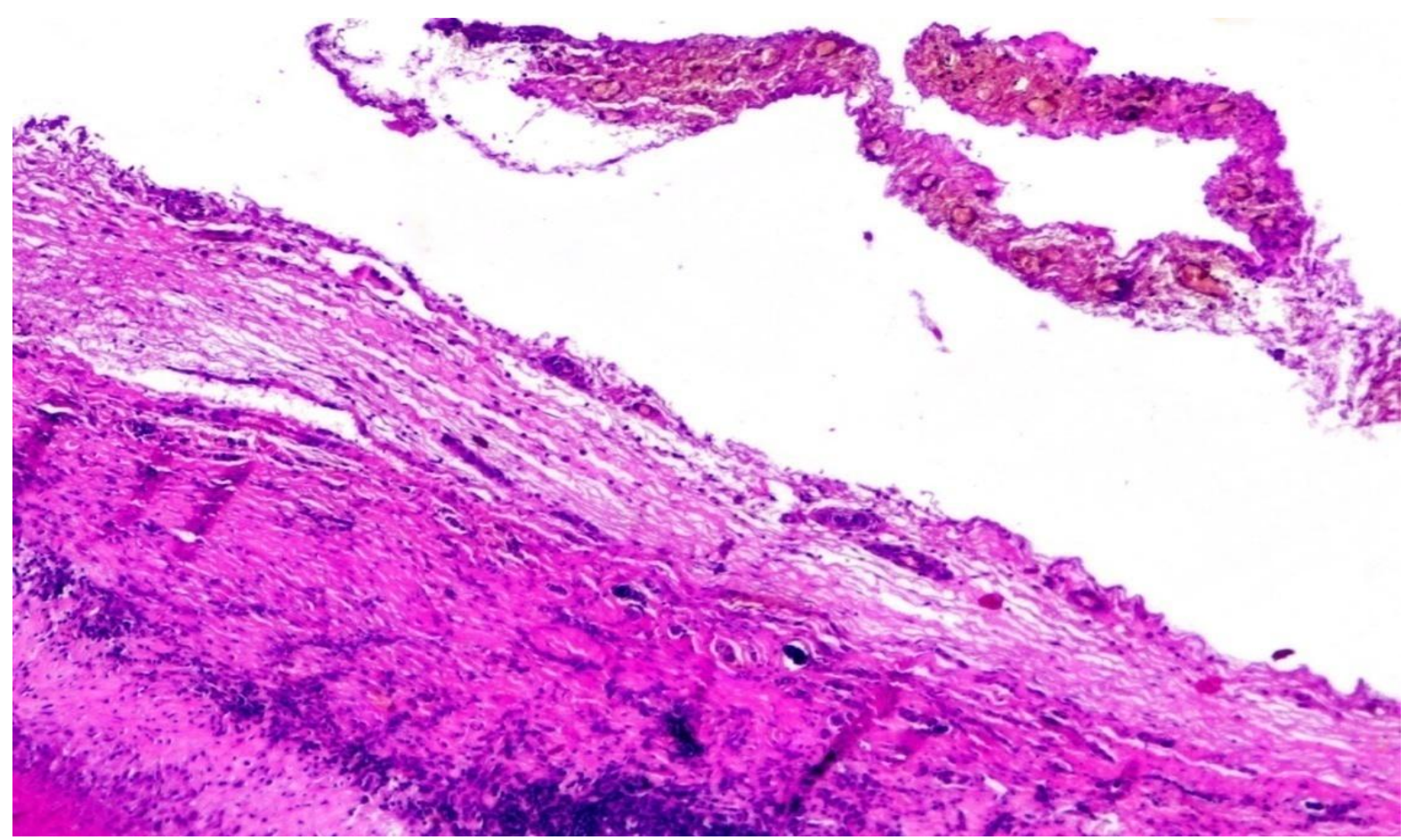

Figure 13:- Loosening of fibrous envelope by the HIFU therapy exposure of the dose rate of $200 \mathrm{~W}$. Histologic section. Tinction by hematoxylin and eosin. Magnification x 100 .

The morphological changes were intensified under exposure of $300 \mathrm{~W}$. Cells of the separated germinal envelope have been vacuolizated according to Figure 14.

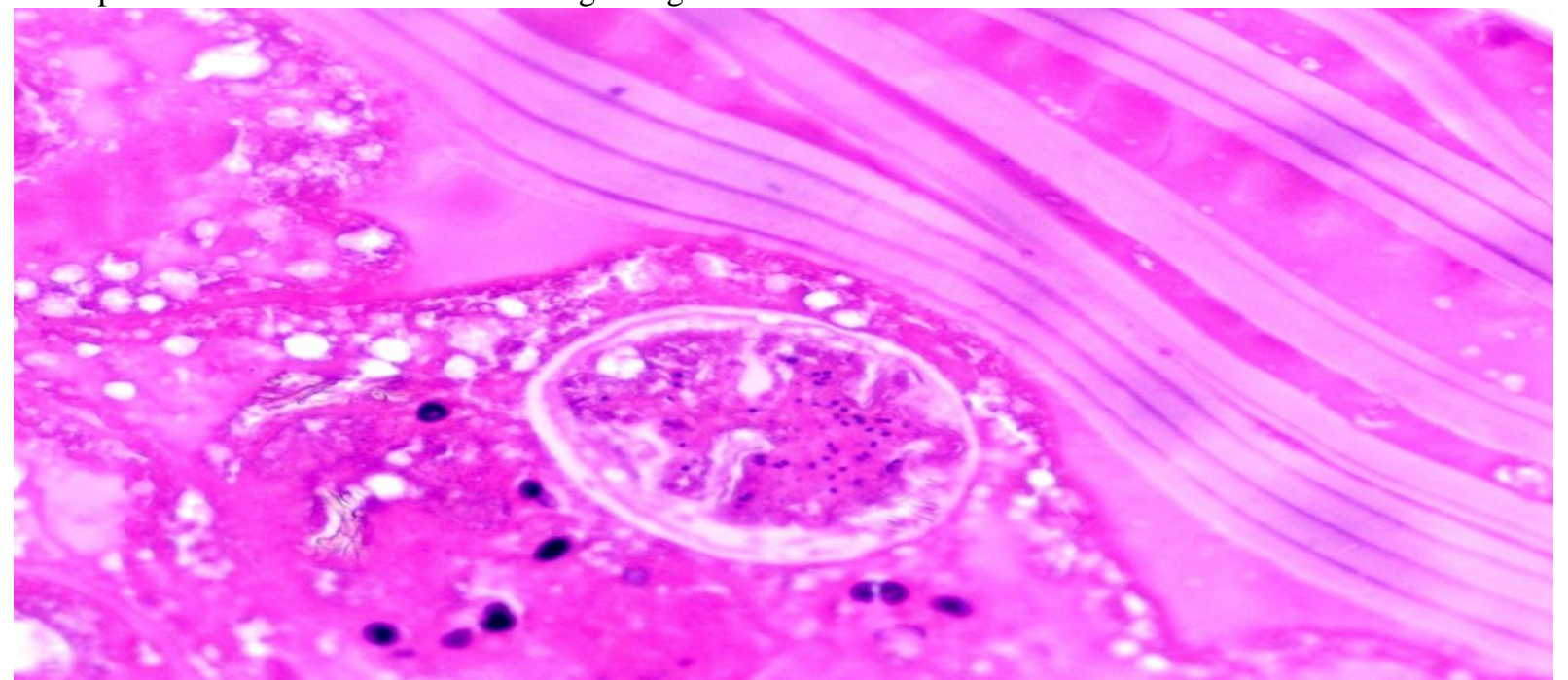

Figure 14:- Vacuolization of the cells of germinal envelope under exposure to the HIFU therapy of the dose rate of $300 \mathrm{~W}$. Histologic section. Tinction by hematoxylin and eosin. Magnification x 100. 
Protoscolexes were exposed to destruction and necrosis according to Figure 15.

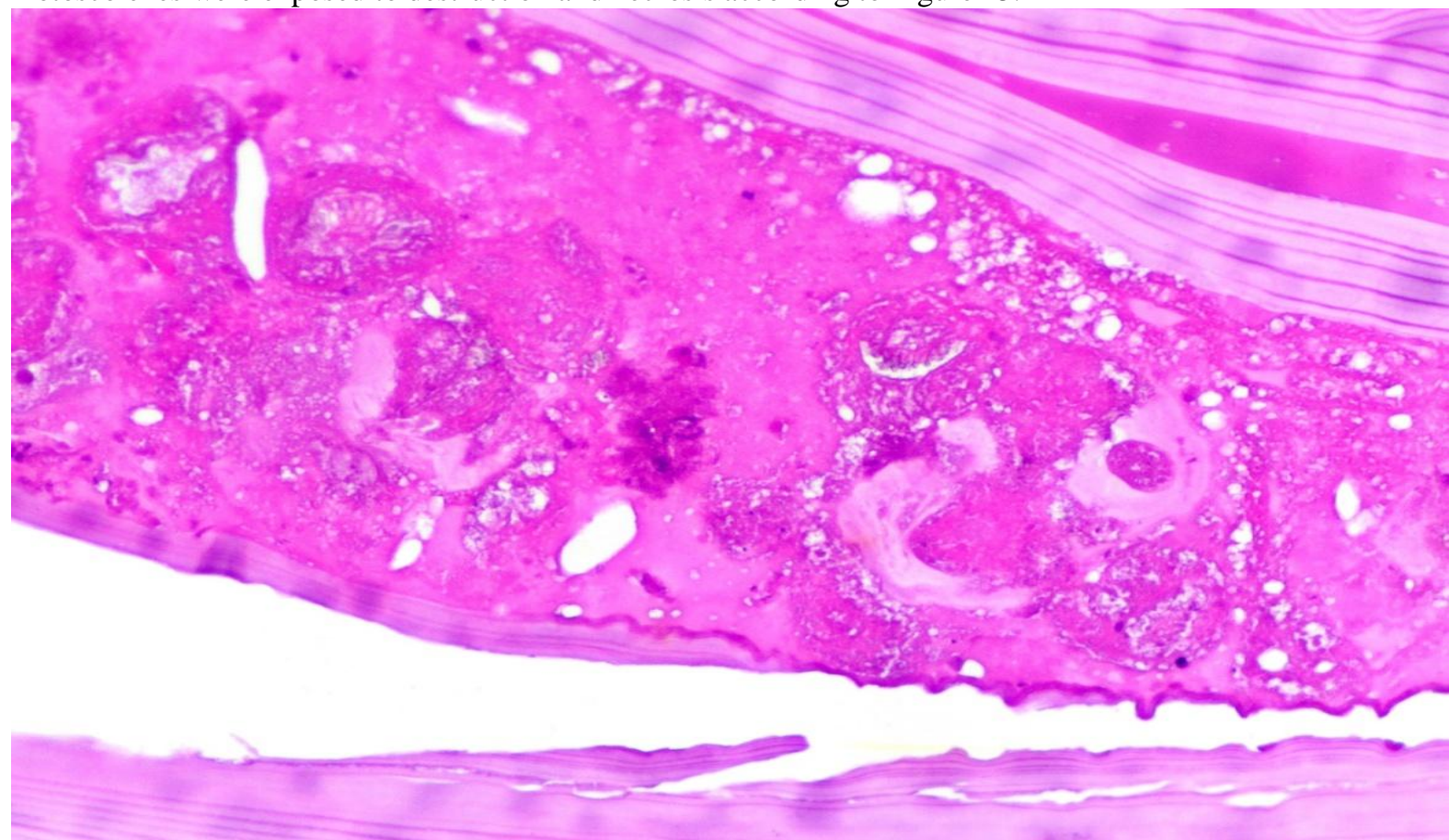

Figure 15:- The protoscolexes necrosis under exposure to the HIFU therapy of the dose rate of 300W. Histologic section. Tinction by hematoxylin and eosin. Magnification x 100.

The chitinized envelope's inner layers, adjacent to the germinal envelope, were homogenized and 'melted'. The fibrous capsule tissue was full-blooded and loosened according to Figure 16.

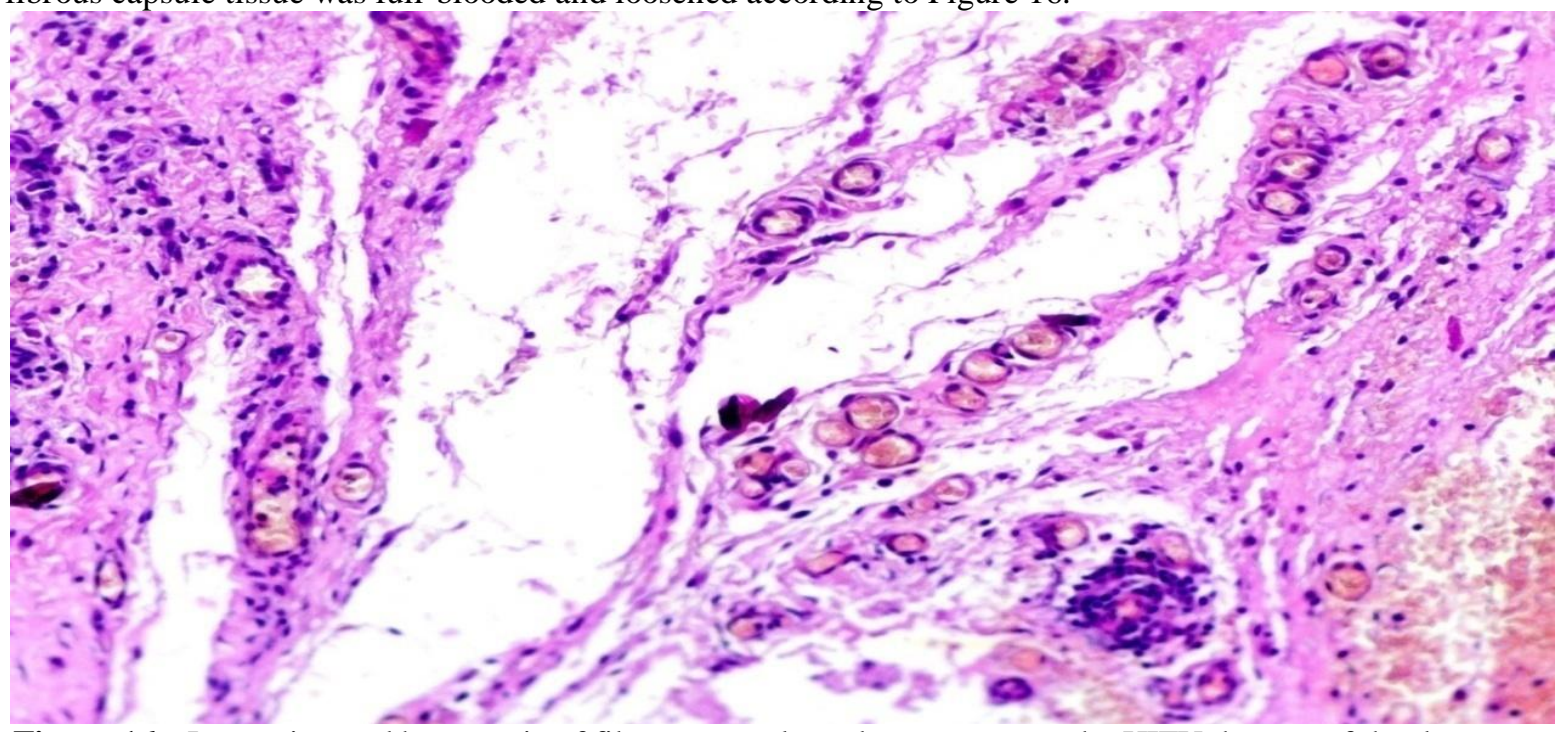

Figure 16:- Loosening and hyperemia of fibrous capsule under exposure to the HIFU therapy of the dose rate of 300W.Histologic section. Tinction by hematoxylin and eosin. Magnification x 100. 
According to Figure 17, only strips of germinal envelope were remained under exposure to the dose rate of 400W.

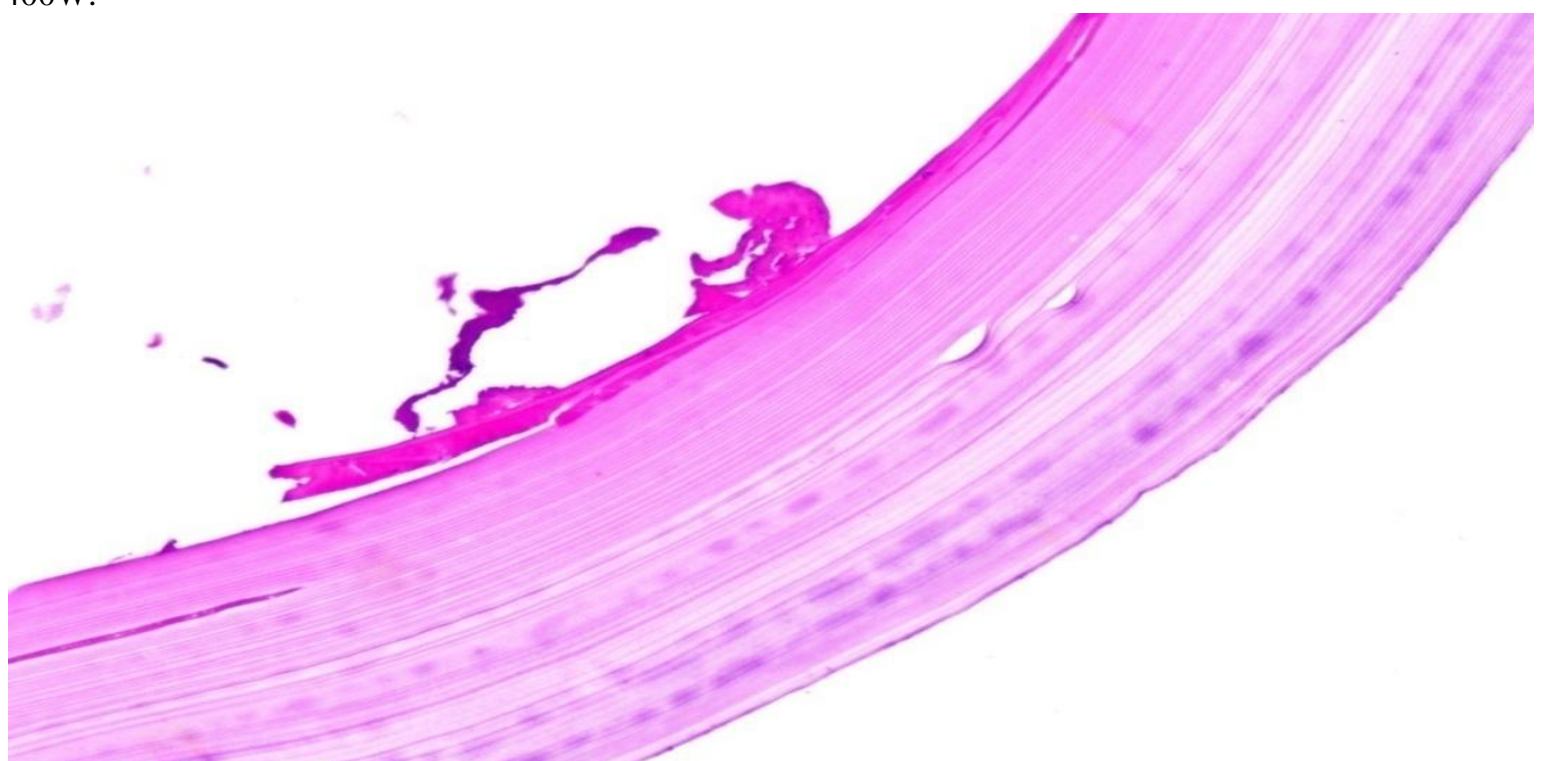

Figure 17:- Strips of the germinal envelope under exposure to the HIFU therapy of the dose rate of 400W. Histologic section. Tinction by hematoxylin and eosin. Magnification x 100.

Due to coagulation the inner layers of chitinized envelope were necrotized, as if they were 'charred', according to Figure 18.

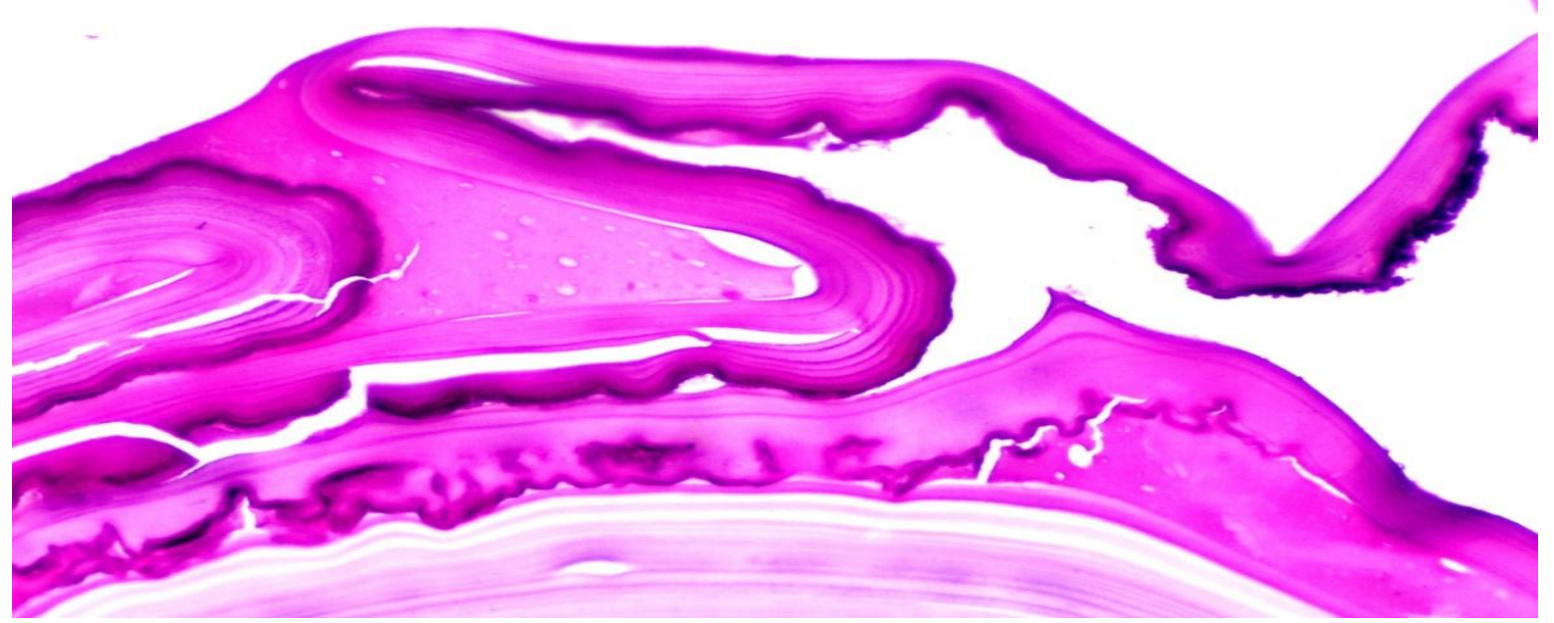

Figure 18:- The chitinized envelope necrosis under exposure to the HIFU therapy of the dose rate of 400W. Histologic section. Tinction by hematoxylin and eosin. Magnification x 100 in spectrum of malignant and benign tumors in palliative and radical term. 
The fibrous capsule was exposed to obvious destruction and necrosis according to Figure 19.

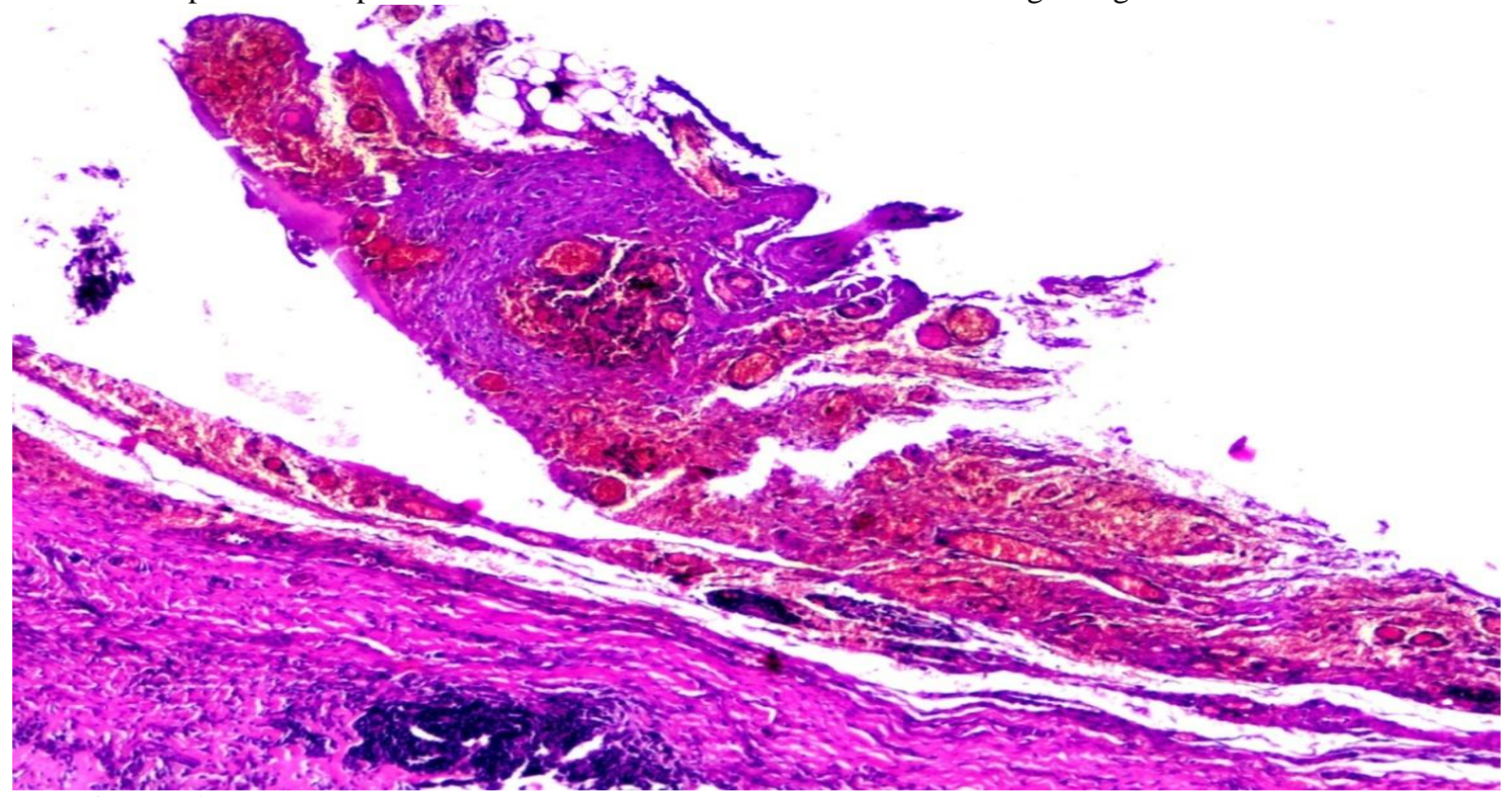

Figure 19:- The fibrous envelope destruction under exposure to the HIFU therapy of the dose rate of 400W.Histologic section. Tinction by hematoxylin and eosin. Magnification x 100.

\section{Conclusion:}

Thus, the minimum capacity of the impact of high-intensity focused ultrasound (HIFU) was the dose of $200 \mathrm{~W}$ which brings the expressed destructive changes of the hydatid Echinicoccus larvocyst structure elements.

\section{References:-}

1. G. H. Mussayev. Hydatid echinococcosis : diagnosis and complex treatment. : Autoref., MD - M., 2000, 38 p.

2. T. V. Lapina. Diagnosis and treatment of recurrent lungs and liver echinococcosis, and combined multiple locations. Diss., MD - Dushanbe, 2004, 222 p.

3. A. I. Mussayev, Zh. O. Belekov, R. M. Bashirov. Diagnosis and surgical treatment of complicated and combined forms of liver echinococcosis.-Bishkek: "Continent", 2004, 254 p.2.

4. S.Imankulov, A.Baigenzhin, T.Tuganbekov, N.Zhampeissov. Hydatid echinococcosis - a modern view. -J Clin Med Kaz 2015; 2(36):11-14.

5. S. Imankulov, G. Fedotovskikh, G. Shaimardanova, M. Yerlan. Feasibility study of high intensity focused ultrasound (HIFU) for the treatment of hydatid cysts of the liver - Elsevier. J.Ultrasonics sonochemistry 27 (2015), 67-75. 